\title{
Current Distribution Optimization in Electromagnet: Application to Superconducting Linear Acceleration System*)
}

\author{
Takazumi YAMAGUCHI ${ }^{1)}$, Teruou TAKAYAMA ${ }^{2)}$, Atsushi KAMITANI ${ }^{2)}$ and Hiroaki OHTANI ${ }^{1,3)}$ \\ 1) The Graduate University for Advanced Studies (SOKENDAI), Toki 509-5292, Japan \\ ${ }^{2)}$ Yamagata University, Yonezawa 992-8510, Japan \\ ${ }^{3)}$ National Institute for Fusion Science, Toki 509-5292, Japan
}

(Received 29 November 2019 / Accepted 31 May 2020)

\begin{abstract}
The current distribution in the electromagnet is optimized so as to maximize the acceleration performance of a superconducting linear acceleration (SLA) system. As a novel pellet injection system for a fusion reactor, the SLA system has been proposed recently. The SLA system is composed of the electromagnet and the pellet container to which a high-temperature superconducting (HTS) film is attached. The pellet container is accelerated by using the Lorentz force between the HTS film and the electromagnet. In the present study, the current distribution in the electromagnet is represented as a set of the filaments by the on-off method. Moreover, the current distribution optimization is performed by using the non-dominated sorting genetic algorithm II (NSGA-II). Furthermore, the dynamic motion of the pellet container is determined by solving the equivalent-circuit model and Newton's equation of motion. According to the numerical results, the acceleration performance is improved by partly applying the electric current to the electromagnet. Compared with the case of the homogeneous current distribution, the pellet velocity for the optimized current distribution increases by $26 \%$.
\end{abstract}

(c) 2020 The Japan Society of Plasma Science and Nuclear Fusion Research

Keywords: accelerator magnet, current distribution optimization, equivalent-circuit model, high-temperature superconductor, NSGA-II, on-off method

DOI: $10.1585 /$ pfr. 15.2405050

\section{Introduction}

A pellet injection for a fusion reactor is required to generate and maintain ultra-high-temperature plasma. In order to maintain the plasma for a long period of time, the pellet must be injected into the core plasma. To this end, it is necessary to increase the pellet velocity to $5 \mathrm{~km} / \mathrm{s}[1,2]$.

As an alternative pellet injection system, a superconducting linear acceleration (SLA) system has been proposed recently. The SLA system is composed of an electromagnet and a pellet container to which a high-temperature superconducting (HTS) film is attached. In the SLA system, the container is accelerated by the electromagnetic force between the HTS film and the electromagnet. Because the experiment of the SLA system has not been performed yet, its acceleration performance has not been clear.

In order to clarify the acceleration performance, we simulated the dynamic motion of the container accelerated by the multiple-electromagnet SLA system. As a result of the SLA simulation [3], it was clear that the pellet velocity can reach up to $5 \mathrm{~km} / \mathrm{s}$. However, a considerable number of the electromagnets must be placed over $40 \mathrm{~km}$. Therefore, it is indispensable to improve the acceleration performance of each electromagnet.

author's e-mail: yamaguchi.takazumi@nifs.ac.jp

*) This article is based on the presentation at the 28th International Toki Conference on Plasma and Fusion Research (ITC28).
The purpose of the present study is to optimize the current distribution so as to maximize the acceleration performance of a single electromagnet.

\section{Governing Equation}

A schematic view of the SLA system is shown in Fig. 1. In this system, a fuel pellet is carried by a pellet container to which two types of HTS film are attached. The container is levitated by the repulsive force between the permanent-magnet rail and the levitation HTS film. Moreover, the acceleration HTS film is exposed to a magnetic flux density generated by an electromagnet. As a result, the container is accelerated by the Lorentz force acting on the acceleration HTS film. Throughout the present study, the shape of the acceleration HTS film is assumed to be the

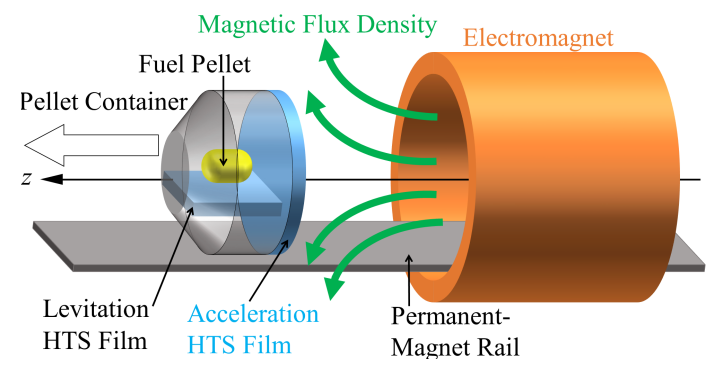

Fig. 1 A schematic view of the SLA system. 


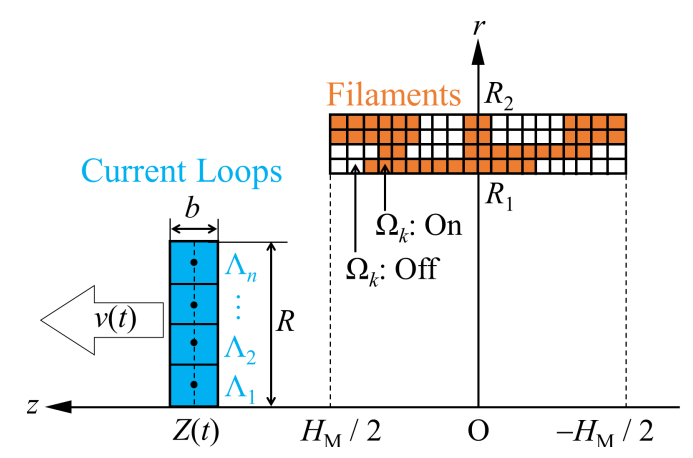

Fig. 2 An equivalent-circuit model. In this figure, the electromagnet is approximated as a set of the filaments for the case with $K_{r}=4$ and $K_{z}=20$.

disk-shaped film of radius $R$ and thickness $b$. In addition, the axisymmetric magnetic flux density is generated by the cylindrical electromagnet of inner radius $R_{1}$, outer radius $R_{2}$, and length $H_{\mathrm{M}}$.

Under the above assumption, the shielding current in the acceleration HTS film and the dynamic motion of the container can be analyzed numerically by using the equivalent-circuit model (ECM) [3,4]. In the ECM, the distribution of the shielding current density is approximated as a set of $n$ current loops, $\Lambda_{1}, \Lambda_{2}, \cdots, \Lambda_{n}$ (See Fig. 2). Here, $\Lambda_{i}$ denotes the $i$ th current loop of radius $r_{i}$ having the cross section of width $\Delta r_{i}$ and thickness $b$. Furthermore, the electric current and the voltage in $\Lambda_{i}$ are denoted by $I_{i}$ and $V_{i}$, respectively. Note that the effects of the levitation HTS film, the container, and the rail are neglected.

On the other hand, the cross section $\left[R_{1}, R_{2}\right] \times$ [ $\left.-H_{\mathrm{M}} / 2, H_{\mathrm{M}} / 2\right]$ of the electromagnet is radially divided in $K_{r}$ and longitudinally divided in $K_{z}$. Here, $K$ is defined by $K \equiv K_{r} K_{z}$. Namely, the electromagnet is approximated as a set of $K$ filaments, $\Omega_{1}, \Omega_{2}, \cdots, \Omega_{K}$ (See Fig. 2 ). As a result, the current distribution in the electromagnet can be represented by the on-off method [5]. In the on-off method, the $k$ th filament $\Omega_{k}$ is called on-state when electric current flows in $\Omega_{k}$. Otherwise, $\Omega_{k}$ is called off-state. Accordingly, the current distribution is represented in terms of a state vector:

$$
\mathbf{x} \equiv\left(x_{1}, x_{2}, \cdots, x_{K}\right)^{\mathrm{T}},
$$

where

$$
x_{k} \equiv\left\{\begin{array}{ll}
1 & \left(\Omega_{k}: \text { on-state }\right) \\
0 & \left(\Omega_{k}: \text { off-state }\right)
\end{array} .\right.
$$

By using $\mathbf{x}$, electric currents of all the filaments are controlled by

$$
\mathbf{I}^{\mathrm{F}}(t, Z) \equiv \frac{I_{\text {total }}(t, Z)}{K} \mathbf{x} .
$$

Here, the $k$ th component of $\mathbf{I}^{\mathrm{F}}(r, Z)$ denotes an electric current in $\Omega_{k}$. In addition, $I_{\text {total }}$ is a total current when all the filaments are on-state. In the present study, $I_{\text {total }}(t, Z)$ is defined by the following equation:

$$
I_{\text {total }}(t, Z) \equiv\left\{\begin{array}{ll}
\alpha t & \left(0 \leq Z \leq Z_{\text {limit }}\right) \\
0 & \text { (otherwise) }
\end{array},\right.
$$

where $\alpha$ is an increasing rate of $I_{\text {total }}(t, Z)$, and the inequality $0 \leq Z \leq Z_{\text {limit }}$ denotes an acceleration range.

By using the ECM and the on-off method, Faraday's law becomes equivalent to the following circuit equation [4]:

$$
L \frac{d \mathbf{I}}{d t}=-\left[\mathbf{M}(Z) \frac{d I_{\mathrm{total}}}{d t}+\frac{d \mathbf{M}}{d Z} v I_{\mathrm{total}}+\mathbf{V}\right],
$$

where $\mathbf{I} \equiv\left(I_{1}, I_{2}, \cdots, I_{n}\right)^{\mathrm{T}}, \mathbf{M}(Z) \equiv M(Z) \mathbf{x} / K$, and $\mathbf{V} \equiv\left(V_{1}, V_{2}, \cdots, V_{n}\right)^{\mathrm{T}}$. Moreover, $L$ is an $n$-by- $n$ inductance matrix, and its $(i, j)$ entry is the inductance by $\Lambda_{j}$ on $\Lambda_{i}$. Furthermore, $M(Z)$ is an $n$-by- $K$ mutual-inductance matrix, and its $(i, k)$ entry is the mutual inductance by $\Omega_{k}$ on $\Lambda_{i}$. In addition, $Z$ and $v(\equiv d Z / d t)$ are the position and the velocity of the HTS film, respectively. In order to determine the induced voltage $V_{i}$ in $\Lambda_{i}$, we adopt the power law [6,7], $V_{i}=V_{\mathrm{C} i}\left(\left|I_{i}\right| / I_{\mathrm{C} i}\right)^{N} \operatorname{sgn}\left(I_{i}\right)$, where $V_{\mathrm{C} i}$ is a critical voltage and is defined by $V_{\mathrm{C} i} \equiv 2 \pi\left(r_{i}+\Delta r_{i} / 2\right) E_{\mathrm{C}}$. Also, $I_{\mathrm{C} i}$ is a critical current and is defined by $I_{\mathrm{C} i} \equiv b \Delta r_{i} j_{\mathrm{C}}$. Here, $E_{\mathrm{C}}$ and $j_{\mathrm{C}}$ denote a critical electric field and a critical current density, respectively. In addition, $N$ is a positive constant.

On the other hand, the dynamic motion of the container is governed by the following Newton's equation of motion:

$$
m \frac{d^{2} Z}{d t^{2}}=-2 \pi \sum_{i=1}^{n} r_{i} B_{r}\left(Z, r_{i}, t\right) I_{i},
$$

where $m$ is the total mass of the container and the HTS film. Moreover, $B_{r}(z, r, t)=\sum_{k=1}^{K} B_{r, k}(z, r, t)$. Here, $B_{r, k}(z, r, t)$ denotes the $r$-component of the magnetic flux density generated by the $k$ th filament, and it is evaluated by means of the Biot-Savart law. Because the container moves in extremely low pressure, air resistance is neglected in the right-hand side of (6).

By solving the initial-value problem of (5) and (6), both the time evolution of the shielding current and the dynamic motion of the container can be determined. Throughout the present study, the initial condition is as follows: $\mathbf{I}(t)=\mathbf{0}, v(t)=0 \mathrm{~m} / \mathrm{s}, Z(t)=Z_{0}$ at $t=0$. Here, $Z_{0}$ denotes an initial position of the HTS film. Moreover, the initial-value problem is solved by means of the 5 th order Runge-Kutta method with the adaptive step-size control algorithm [8].

\section{Optimization Method}

In order to evaluate the acceleration performance of the SLA system, we use a speedup ratio and an on-ratio. The speedup ratio $f_{\mathrm{v}}$ is defined by the following equation:

$$
f_{\mathrm{v}}(\mathbf{x}) \equiv \frac{v_{\mathrm{f}}(\mathbf{x})}{v_{\mathrm{ref}}},
$$




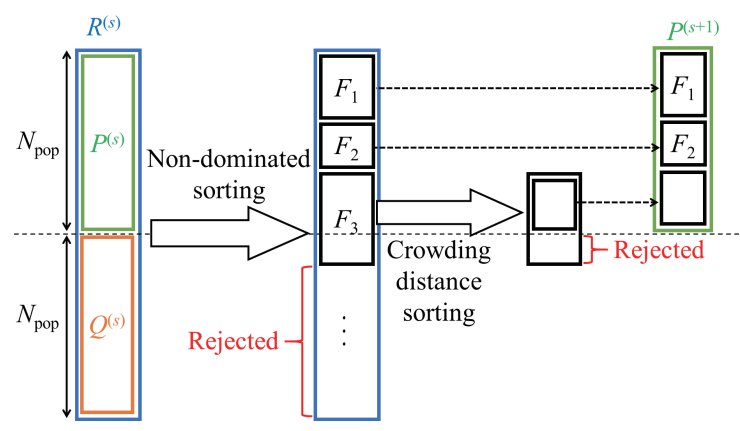

Fig. 3 A procedure of the NSGA-II.

where $v_{\mathrm{f}}(\mathbf{x})$ is the final velocity for the current distribution obtained by $\mathbf{x}$, and the final velocity is velocity $v(t)$ at the time when $Z(t)=Z_{\text {limit }}$ is satisfied. Also, $v_{\text {ref }}$ is the final velocity for the homogeneous current distribution. The velocities $v_{\mathrm{f}}(\mathbf{x})$ and $v_{\text {ref }}$ are determined by using the ECM. On the other hand, the on-ratio $f_{\text {on }}$ is defined by the following equation:

$$
f_{\text {on }}(\mathbf{x}) \equiv \frac{K_{\text {on }}(\mathbf{x})}{K},
$$

where $K_{\mathrm{on}}(\mathbf{x})$ is the number of the on-state filaments. The state vector $\mathbf{x}$ is optimized so as to maximize $f_{\mathrm{v}}(\mathbf{x})$ and to minimize $f_{\text {on }}(\mathbf{x})$.

As an optimization method, we adopt the Nondominated Sorting Genetic Algorithm II (NSGA-II) [9]. Because the NSGA-II can maximize or minimize multiple objective functions without introducing new parameter, it is effective for multiobjective optimization problem. In addition, the NSGA-II can be applied to both the maximization and the minimization of the objective functions. The procedure of the NSGA-II is shown as follows:

1. Initial parent population $P^{(1)}$ is generated randomly, and $f_{\mathrm{v}}(\mathbf{x})$ and $f_{\text {on }}(\mathbf{x})$ for $\mathbf{x} \in P^{(1)}$ are calculated. Here, the parent population $P^{(s)}$ is defined as a set of $N_{\text {pop }}$ state vectors. Moreover, the superscript $(s)$ denotes generation.

2. After offspring population $Q^{(s)}$ is produced by the single-point crossover, $f_{\mathrm{v}}(\mathbf{x})$ and $f_{\text {on }}(\mathbf{x})$ for $\mathbf{x} \in Q^{(s)}$ are calculated. Here, the size of $Q^{(s)}$ is $N_{\text {pop }}$.

3. Combined population $R^{(s)}\left(\equiv P^{(s)} \cup Q^{(s)}\right)$ is roughly divided into subset, $F_{1}, F_{2}, \cdots$, by means of the nondominated sorting [9] (See Fig. 3). The subset $F_{i}$ is composed of state vectors having the same acceleration performance.

4. The acceleration performance of state vectors in $F_{i}$ are appreciated by using the crowding distance sorting [9]. By using these sorting, the new parent population $P^{(s+1)}$ is produced. For the case of Fig. 3, $P^{(s+1)}$ is composed of $F_{1}, F_{2}$, and the part of $F_{3}$.

5. Steps $2-4$ are iterated until the termination condition, $s=s_{\max }$, is satisfied.

In the present study, the fastest state vector in $F_{1}$ at $s=$ $s_{\max }$ is regarded as the optimized solution.

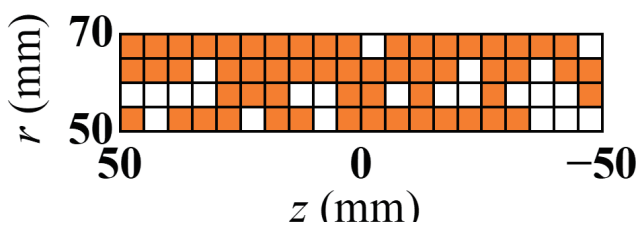

Fig. 4 Optimized current distribution. In this figure, the orange and the white squares show the on-state and the off-state, respectively.

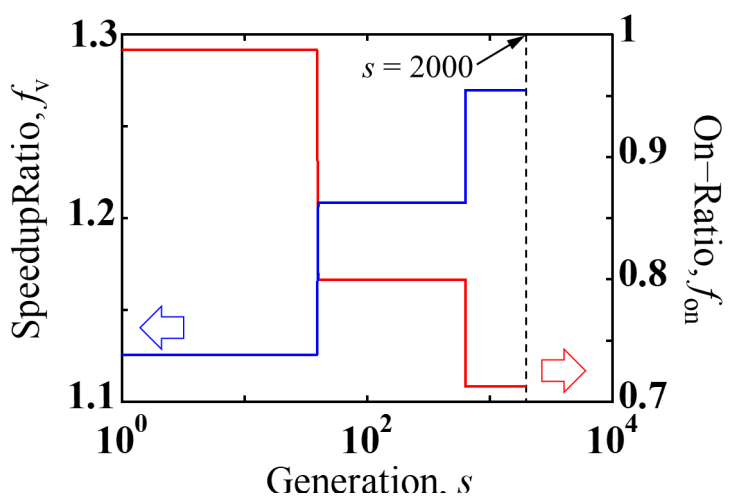

Fig. 5 Dependence of the speedup ratio $f_{\mathrm{v}}$ and the on-ratio $f_{\text {on }}$ on the generation $s$.

\section{Numerical Result}

On the basis of the NSGA-II with the on-off method, we optimize the current distribution in the electromagnet so as to maximize the acceleration performance. In addition, we numerically investigate the acceleration performance for the optimized current distribution. The geometrical and the physical parameters are fixed as follows: $R=40 \mathrm{~mm}, b=1 \mathrm{~mm}, E_{\mathrm{C}}=1 \mathrm{mV} / \mathrm{m}, j_{\mathrm{C}}=10 \mathrm{kA} / \mathrm{mm}^{2}$, $N=20, m=10 \mathrm{~g}, Z_{0}=1 \mathrm{~mm}, R_{1}=50 \mathrm{~mm}, R_{2}=70 \mathrm{~mm}$, $H_{\mathrm{M}}=100 \mathrm{~mm}, \alpha=20 \mathrm{kA} / \mathrm{ms}$, and $Z_{\text {limit }}=300 \mathrm{~mm}$. In addition, the number of the current loops $n$ is $n=100$. Moreover, $K_{r}=4$ and $K_{z}=20$. Namely, the number of the filaments $K$ is $K=80$. Furthermore, the population size $N_{\text {pop }}$ and the maximum number $s_{\max }$ of the iterations are $N_{\text {pop }}=100$ and $s_{\max }=2000$, respectively.

Firstly, let us perform the current distribution optimization by using the NSGA-II. The optimized current distribution is shown in Fig. 4. It is clear from this figure that the electric current is partly applied to the electromagnet. Figure 5 shows the dependence of the speedup ratio $f_{\mathrm{v}}$ and the on-ratio $f_{\text {on }}$ for the optimized solution on the generation $s$. As shown in this figure, the speedup ratio $f_{\mathrm{v}}$ increases with generation $s$. In addition, the on-ratio $f_{\text {on }}$ decreases with an increase in generation $s$. The speedup ratio $f_{\mathrm{v}}$ and the on-ratio $f_{\text {on }}$ finally reach to 1.26 and 0.71 , respectively. In other words, the final velocity increases by $26 \%$, and the cross section in which the electric current flows decreases to $71 \%$. The time dependence of the velocity $v(t)$ is shown in Fig. 6. It is clear from this figure that the velocity $v(t)$ is 


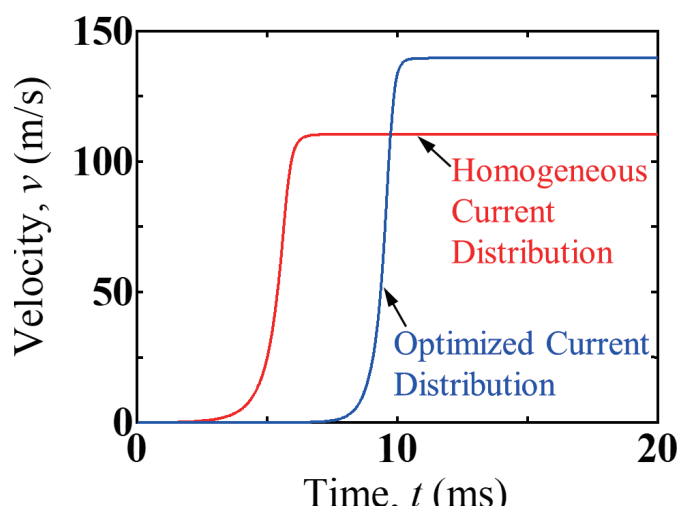

Fig. 6 Time dependence of the velocity $v(t)$.

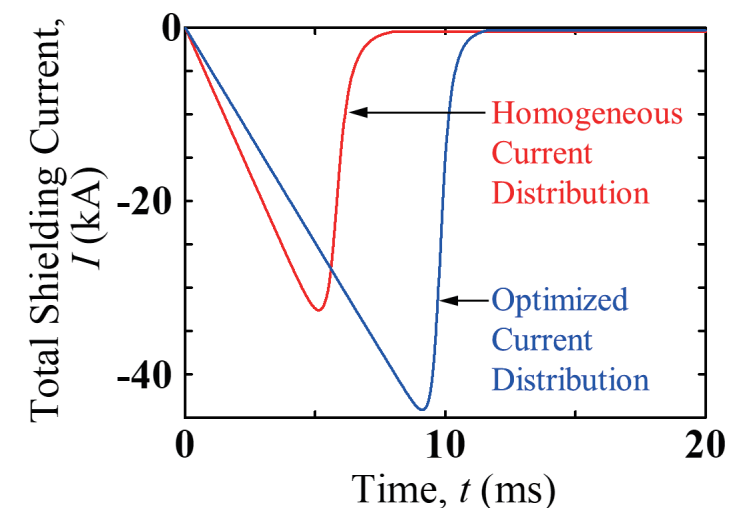

Fig. 7 Time dependence of the total shielding current $I(t)$.

increased from $110 \mathrm{~m} / \mathrm{s}$ to $140 \mathrm{~m} / \mathrm{s}$ by the current distribution optimization.

Next, let us investigate the cause of improving the acceleration performance. According to (6), the velocity $v(t)$ depends on the shielding current $I_{i}(i=1,2, \cdots, n)$ and the $r$-component $B_{r}$ of the magnetic flux density. The time dependence of the total shielding current $I(t)$ is shown in Fig. 7, where $I(t) \equiv \sum_{i=1}^{n} I_{i}(t)$. As shown in this figure, the amount $|I(t)|$ of the total shielding current is increased by the current distribution optimization. Namely, the current distribution in the electromagnet is optimized so that the shielding current density flows increasingly in the HTS film. On the other hand, the dependence of the magnetic flux density $B_{r}(Z(t), R)$ on the film position $Z(t)$ is shown in Fig. 8. This figure shows that the $r$-component $B_{r}(Z(t), R)$ of the magnetic flux density is increased by the optimization. Since both the amount $|I|$ of the total shielding current and the $r$-component $B_{r}$ of the magnetic flux density increase, the acceleration performance is improved.

\section{Conclusion}

By using the on-off method and the NSGA-II, we have optimized the current distribution in the electromagnet so as to maximize the acceleration performance of the SLA

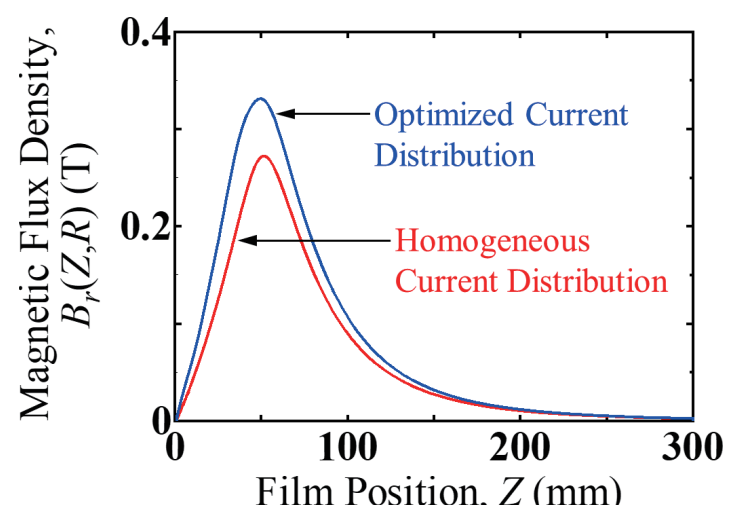

Fig. 8 Dependence of the magnetic flux density $B_{r}(Z, R)$ on the film position $Z$. Here, $R$ denotes the film radius.

system. Conclusions obtained by the present study are summarized as follows:

1. By partly applying the electric current to the electromagnet, the acceleration performance of the SLA system is improved. For the case with the optimized current distribution, the cross section to which the electric current is applied reduced to $71 \%$.

2. Compared with the case of the homogeneous current distribution, the pellet velocity for the optimized current distribution increases by $26 \%$. Therefore, the current distribution optimization might contribute the development of the SLA system.

\section{Acknowledgment}

This work is performed with the support and under the auspices of the NIFS Collaboration Research program (NIFS18KKGS023, NIFS19KNSS127). Moreover, the numerical computations were carried out on Fujitsu PRIMERGY RX4770 M2 of the Data Analysis Server in NIFS.

[1] P.B. Parks and R.J. Turnbull, Phys. Fluids 21, 1735 (1978).

[2] S.L. Milora and C.A. Foster, IEEE Trans. Plasma Sci. 6, 578 (1978).

[3] T. Yamaguchi, T. Takayama, A. Saitoh and A. Kamitani, IEEE Trans. Magn. 55, 7204305 (2019).

[4] T. Yamaguchi, T. Takayama, A. Saitoh and A. Kamitani, J. Adv. Simulat. Sci. Eng. 4, 209 (2017).

[5] Y. Hidaka, T. Sato and H. Igarashi, IEEE Trans. Magn. 50, 7015204 (2014).

[6] E.H. Brandt, Phys. Rev. B 54, 4246 (1996).

[7] A. Kamitani, T. Takayama and S. Ikuno, IEEE. Trans. Magn. 44, 926 (2008).

[8] W.H. Press, S.A. Teukolsky, W.T. Vetterling and B.P. Flannery, Numerical Recipes in Fortran 77, 2nd ed. (Cambridge University Press, New York, 1992) Chap. 16.

[9] K. Deb, A. Pratap, S. Agarwal and T. Meyarivan, IEEE Trans. Evol. Comput. 6, 182 (2002). 